\title{
REVISITANDO AS HEURÍSTICAS DE AVALIAÇÃO DE NIELSEN PARA ANÁLISE DE USABILIDADE EM JOGOS DE TABULEIRO NÃO VIRTUAIS
}

\author{
Allan Kássio Beckman Soares da Cruz \\ Universidade Federal do Maranhão \\ allankassio@gmail.com \\ Carlos de Salles Soares Neto \\ Universidade Federal do Maranhão \\ csallesneto@gmail.com
}

\begin{abstract}
Resumo: As heurísticas de Nielsen foram originalmente propostas e desenvolvidas para avaliação de usabilidade em interfaces de sistemas computacionais. Este trabalho propõe uma análise e redesenho das heurísticas de Nielsen de forma a especializá-las para emprego na avaliação de jogos de tabuleiros não virtuais. As heurísticas foram redesenhadas a partir de levantamento bibliográfico, considerações e modelos gerados sobre as regras encontradas em cada jogo testado, além da análise heurística das interfaces existentes nesses jogos através de método de inspeção utilizando as heurísticas de Nielsen. O objetivo do trabalho visa através desse levantamento, gerar uma base onde seja possível expor a aplicabilidade de tais heurísticas especializadas em ambientes não computacionais e que elas possam ser aplicadas em jogos de tabuleiro distintos dos analisados inicialmente.
\end{abstract}

Palavras-chave: usabilidade, heurísticas de Nielsen, jogo de tabuleiro.

\section{INTRODUÇÃO}

A análise heurística é uma das técnicas de avaliação de usabilidade, mais importantes, e certamente uma das mais fáceis, de aplicar. Ela consiste, basicamente, em submeter a interface de um determinado sistema computacional à avaliação de alguns especialistas em usabilidade, conforme um conjunto previamente determinado de "bons princípios de usabilidade" (NIELSEN, 1994). Denomina-se esses princípios de heurísticas, sendo o principal conjunto destas o criado por Jakob Nielsen, que os expôs no livro Usability Engineering (1993).

\subsection{Preparação da Análise Heurística}

Para se realizar uma análise heurística, precisamos de especialistas em usabilidade, um protótipo do aplicativo (seja em papel, wireframe, implementação 
inicial, etc.), hipóteses iniciais sobre os usuários e bateria de atividades (NIELSEN e MOLICH, 1990).

Os dois primeiros itens são obrigatórios, afinal, não há avaliação ou teste sem o sujeito (especialistas) e o objeto (protótipo do sistema). Por permitir protótipos nãofuncionais e funcionais, essa análise pode ser utilizada em qualquer estágio do ciclo de desenvolvimento. Sendo assim, ela serve para eliminar erros conceituais (isto é, vindos de uma interpretação errada dos requisitos) já na fase inicial do desenvolvimento (NIELSEN e LANDAUER, 1993). Isso evita que esses erros sejam detectados depois, quando a implementação já está adiantada, e a correção deles exige uma remodelagem do sistema, gerando retrabalho.

As hipóteses iniciais sobre os usuários são opcionais, mas mesmo assim são itens importantes a serem aplicados na análise heurística. Pois para uma interface ser fácil e agradável de ser usada, ela deve levar em conta as necessidades dos usuários. Atualmente, são muitos os perfis possíveis, cada um com características próprias de aprendizado, expectativas em relação ao aplicativo, entre outros itens (NIELSEN, 1992). Logo, é desejável que o usuário seja conhecido, para que as interfaces possam atender as necessidades e desejos desse usuário, para que o produto criado possua boas possibilidades comerciais. Assim, as hipóteses iniciais são instrumentos adequados para serem utilizados durante toda a análise heurística (NIELSEN e MOLICH, 1990).

Em relação à bateria de atividades, esta será importante para que o especialista tenha um foco ao realizar a análise heurística. A bateria é composta, preferencialmente, por duas classes de tarefas:

- Tarefas que os usuários-alvo executariam mais frequentemente dentro do aplicativo;

- Tarefas que poderiam apresentar problemas para a compreensão e execução do usuário.

Para que essas tarefas sejam corretamente modeladas, é importante que as hipóteses sobre os usuários tenham sido corretamente estabelecidas (NIELSEN, 1994). Elas é que irão guiar toda a criação das tarefas, visto que nas hipóteses se estabelecem o perfil de uso e as possíveis limitações do usuário (ZHANG et al., 1999).

\subsection{Execução da Análise Heurística}

Depois da fase de preparação, vem a fase de execução da análise heurística. Essa execução se dá em três fases distintas (NIELSEN, 1990):

- Análise individual: nessa fase, cada um dos especialistas analisa individualmente a interface do aplicativo, por um período variável de tempo (usualmente 1-2 horas), segundo o conjunto de heurísticas escolhido. Um relatório é gerado nessa fase, mostrando cada um dos erros encontrados, indicando em cada um a heurística violada, o local do erro e a gravidade do problema, além das possíveis soluções imaginadas pelo especialista.

- Consolidação da análise: nessa fase, todos os especialistas, juntamente com o líder da equipe, reúnem-se para discutir a respeito dos resultados individuais encontrados. Nessa consolidação, cada especialista tem acesso a todos os relatórios individuais gerados na primeira fase. Ao final desta fase, deve ser gerado um relatório unificado, com todos os erros encontrados (da mesma maneira que na primeira fase). 
- Reunião final: nessa fase, os especialistas se reúnem com o cliente lou o gerente de projeto) para definir quais serão os erros de interface a serem corrigidos.

\subsection{Heurísticas}

Existem diversas heurísticas que podem ser utilizadas seguindo os procedimentos relatados anteriormente. Há alguns conjuntos de heurísticas mais utilizados pelos especialistas em usabilidade; são eles: as heurísticas de Bastien e Scapin (1995) e as heurísticas de Nielsen (1993).

As heurísticas de Bastien e Scapin são voltadas para a área da Ergonomia. As heurísticas de Nielsen, por sua vez, cobrem todos os aspectos das boas práticas de usabilidade, sendo usadas quase universalmente, na academia e na indústria.

\section{AS HEURÍSTICAS DE NIELSEN}

Essas heurísticas foram originalmente desenvolvidas por Nielsen para avaliação de usabilidade de interfaces computacionais, em colaboração com Rolf Molich em 1990 (MOLICH \& NIELSEN 1990). E desde então vem sendo refinadas por ele com base em uma análise fatorial de 249 problemas de usabilidade (NIELSEN, 1992) o que permitiu derivar um conjunto de heurísticas com um potencial máximo de utilização e resulta no conjunto revisto de heurísticas a seguir (NIELSEN, 1993).

Visibilidade de status do sistema: a interface do sistema deve sempre informar ao usuário o que está acontecendo. Isto significa que o usuário não pode ficar exposto a uma operação ou interface que não retorne resposta sobre o processo que está ocorrendo e a etapa em que está. Em geral, 10 segundos é o tempo limite para que o usuário mantenha sua atenção no sistema. Neste sentido, é importante sempre dialogar com o usuário utilizando de interfaces apropriadas (como barras de progresso, caixas de mensagem e outras, de preferência com símbolos universais e de fácil entendimento), entender as informações que ele necessita do sistema comunicando estas mensagens em uma linguagem adequada e construir um sistema que forneça elementos que digam ao usuário o que o sistema está continuamente fazendo.

Relacionamento entre a interface do sistema e o mundo real: o sistema deve ser especificado na linguagem do usuário e não fazer uso de linguagem técnica. Isto significa que deve-se ter em mente o tipo médio de usuário que utilizará o sistema e contextualizar a comunicação do sistema ao modelo mental deste tipo de usuário. Em alguns casos, pode ser recomendado ter opções de sistema para habilitar interfaces distintas para diferentes tipos de usuários.

Liberdade e controle do usuário: o usuário, sempre que desejar, deve poder cancelar uma tarefa ou retornar ao ponto anterior. O sistema não pode impedir uma operação do usuário. Caso seja necessário executar uma determinada ação até o final do seu processamento sem interrupção, o sistema deve informar ao usuário os motivos pelos quais a tarefa não pode ser cancelada.

Consistência e padronização: o sistema sempre deve utilizar o mesmo padrão de ícones, símbolos e de palavras. Um mesmo comando ou ação sempre deve ter o mesmo efeito no sistema, independentemente de onde estejam e deve estar sempre na mesma posição. Os códigos de cores, botões básicos e layout, de preferência, devem estar de acordo com o padrão do sistema operacional corrente. 
Prevenção de erros: deve-se criar mecanismos que possam prevenir os erros mais básicos do usuário. Para isto, utiliza-se mensagens antes de operações que possam alterar o sistema para um estado não adequado (por exemplo, ao deletar um arquivo), definir formatos obrigatórios de campos, utilizar campos de preenchimento automático para evitar a digitação errada.

Reconhecimento e não lembrança: sempre que possível, evite que o usuário tenha que lembrar um comando específico. Ofereça elementos de diálogo que permita que o usuário manipular o sistema, mas sem sobrecarregar sua capacidade de memorização.

Flexibilidade e eficiência de uso: o sistema deve ser fácil para uso por usuários comuns, mas deve ser flexível para permitir que usuários avançados possam ter ganho de desempenho. Isto significa que, em um bom sistema, deve-se ter opções diversas para acessar uma mesma funcionalidade. Um exemplo de flexibilidade que pode ser adotada é o uso de teclas de atalhos para algumas funções.

Estética e design minimalista: o texto e o design do sistema devem ser sempre simples e objetivos. Deve-se evitar colocar na interface mais ou menos do que o usuário deve saber.

Ajudar os usuários a reconhecer, diagnosticar e sanar erros: as mensagens de erro do sistema devem ser simples e informar de forma correta ao usuário, além de indicar possíveis soluções claras. A mensagem de erro nunca pode intimidar ao usuário.

Ajuda e documentação: um sistema eficiente deve ser tão fácil de utilizar que o usuário não precise de maior ajuda. Ainda assim, deve ser construído um bom conjunto de documentação e ajuda que seja facilmente acessado pelo usuário em caso de dúvida.

\section{MODELAGEM DA INTERAÇÃO}

O primeiro problema encontrado para a proposta de redesenho das heurísticas é a característica não virtual do sistema na qual as heurísticas serão aplicadas. Por isso são necessárias algumas adaptações propostas pelo autor. Essas adaptações são fundamentadas na bibliografia existente e na inspeção feita pelo autor das regras e dos tabuleiros de jogo.

Todo jogo deverá ser tratado como um sistema propriamente dito, ou seja, deverá possuir entrada, processamento baseado em funções e saída (JEFFRIES et al, 1991). Essa premissa também pode ser aplicada para jogos de tabuleiro não virtuais, ou para qualquer tipo de jogo. Para isso será necessário criar um modelo conceitual do jogo, que deverá possuir as classes e entidades existentes, além de suas interações. 0 tipo de modelagem utilizada foi a baseada no modelo Modeling Language for Interactions as Conversation - MoLIC (BARBOSA \& SANTANA, 2010). Esse modelo foi projetado para auxiliar os designers no planejamento da interação de sistemas. $O$ MoLIC permite representar a interação humano-computador como o conjunto de conversas que os usuários podem ter com o sistema para atingir seus objetivos.

Porém a simples utilização do MoLIC não é suficiente para a aplicação das heurísticas. Isso gera a necessidade do redesenho das mesmas de forma a se adaptar ao processo (DESURVIRE et al, 2004). Antes da proposta do redesenho é importante conhecer os jogos que foram utilizados na pesquisa, bem como os diagramas MoLIC criados para cada um desses jogos. 


\subsection{Jogos utilizados}

Nesse trabalho, os jogos iniciais estudados foram o Xadrez, o Gamão, o Monopoly ${ }^{\circledR}$ e o Risk ${ }^{\circledR}$. Esses jogos foram escolhidos devido a heterogeneidade de peças, tabuleiro, regras e situações possíveis durante o jogo, além de serem facilmente reconhecidos mesmo por pessoa que não tenham o hábito de jogar.

O tabuleiro de Xadrez utilizado segue o padrão clássico com numerações de 1 a 8 em suas linhas e $\mathrm{A}$ à $\mathrm{H}$ em suas colunas, com casas pretas e brancas. As peças possuem um padrão clássico e são feitas de plástico em cores pretas e brancas (FEITOSA, 2007). O tabuleiro de Gamão utilizado também segue o padrão clássico, com 24 casas triangulares de cores preta e branco intercaladas começando com o branco à direita com 15 peças para cada lado nas cores pretas e brancas, além de dois dados de jogo de seis lados (BELMIRO, 1984). Além dos tabuleiros básicos de Monopoly ${ }^{\circledR}$ (HASBRO, 1999) e Risk ${ }^{\circledR}$ (HASBRO, 1999).

A análise foi feita apenas pelo autor, como forma de iniciar um piloto para um trabalho posterior, onde serão feitas observações e grupos de foco com jogadores imersos em sessões de jogos de tabuleiro.

\subsection{Diagramas MoLIC}

Abaixo são apresentados os diagramas MoLIC desenvolvidos para cada um dos jogos citados anteriormente de acordo com suas regras. Esses diagramas foram utilizados como guias de interação para testar cada uma das heurísticas de Nielsen. A Figura 1 mostra o diagrama para o jogo de Xadrez. A Figura 2 mostra o diagrama para o jogo de Gamão. A Figura 3 apresenta o diagrama para o jogo Monopoly ${ }^{\circledR}$ e a Figura 4 para o jogo Risk ${ }^{\circledR}$.

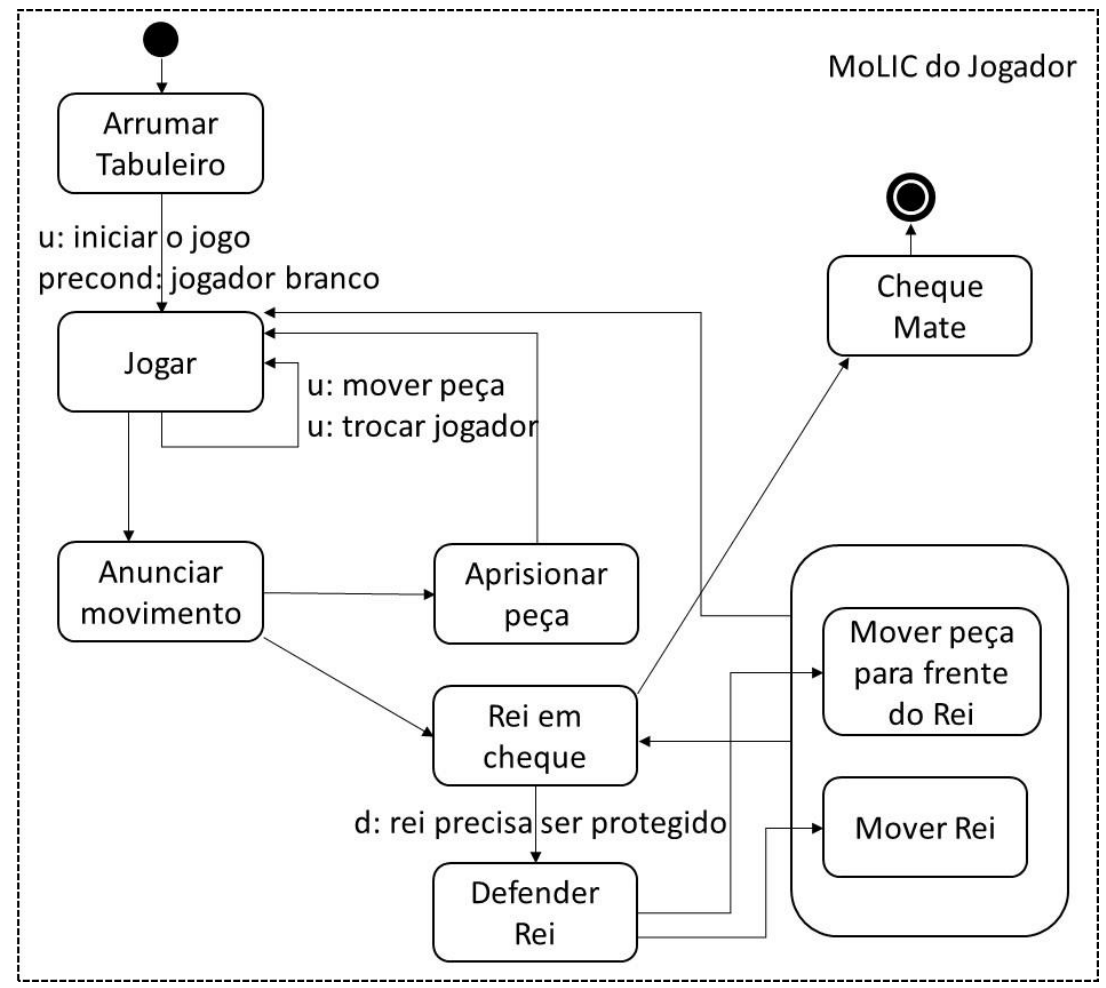

Figura 1 - MoLIC do Xadrez.

Fonte: Elaborado pelo autor, com base na pesquisa realizada. 


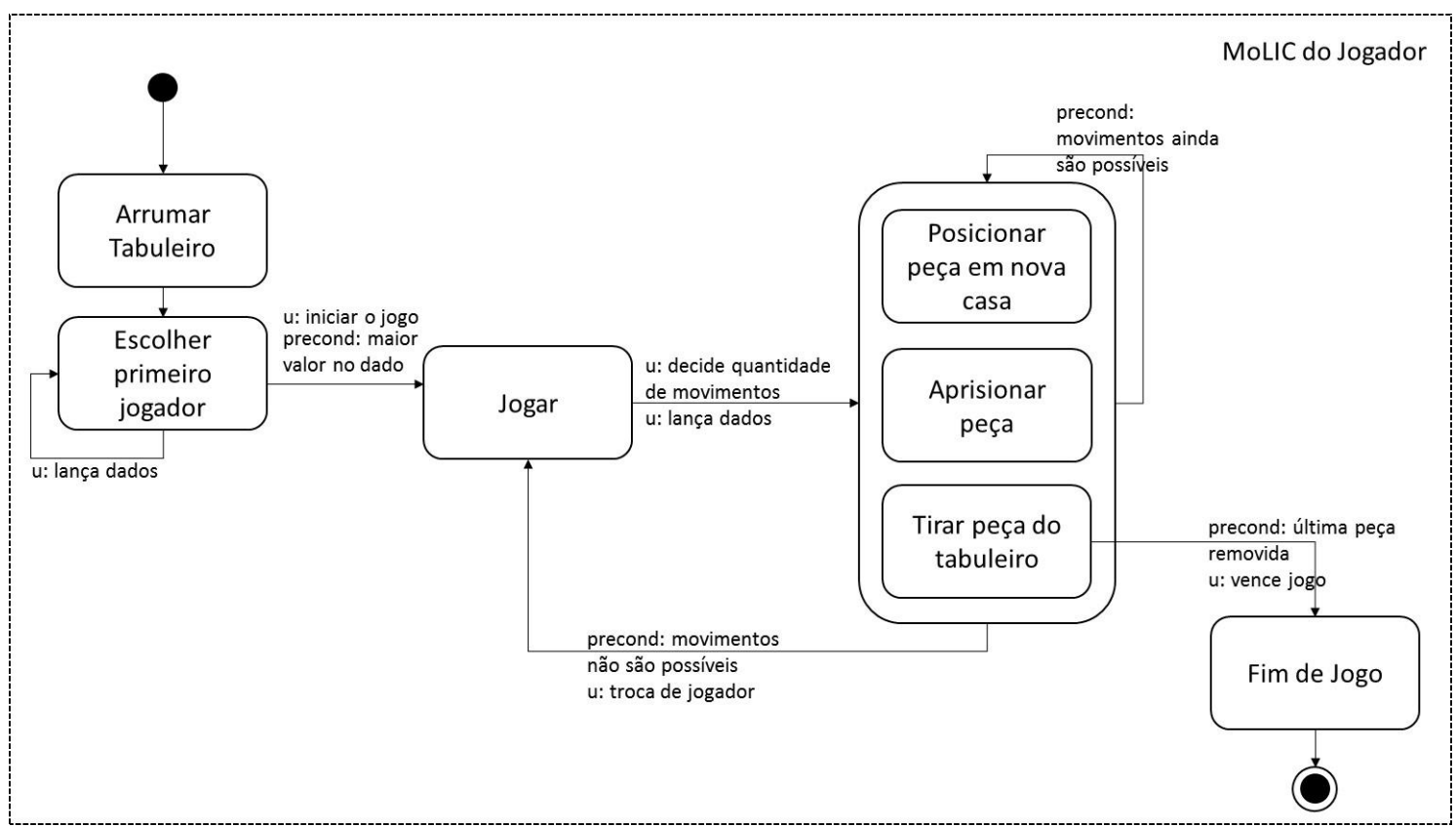

Figura 2 - MoLIC do Gamão.

Fonte: Elaborado pelo autor, com base na pesquisa realizada.

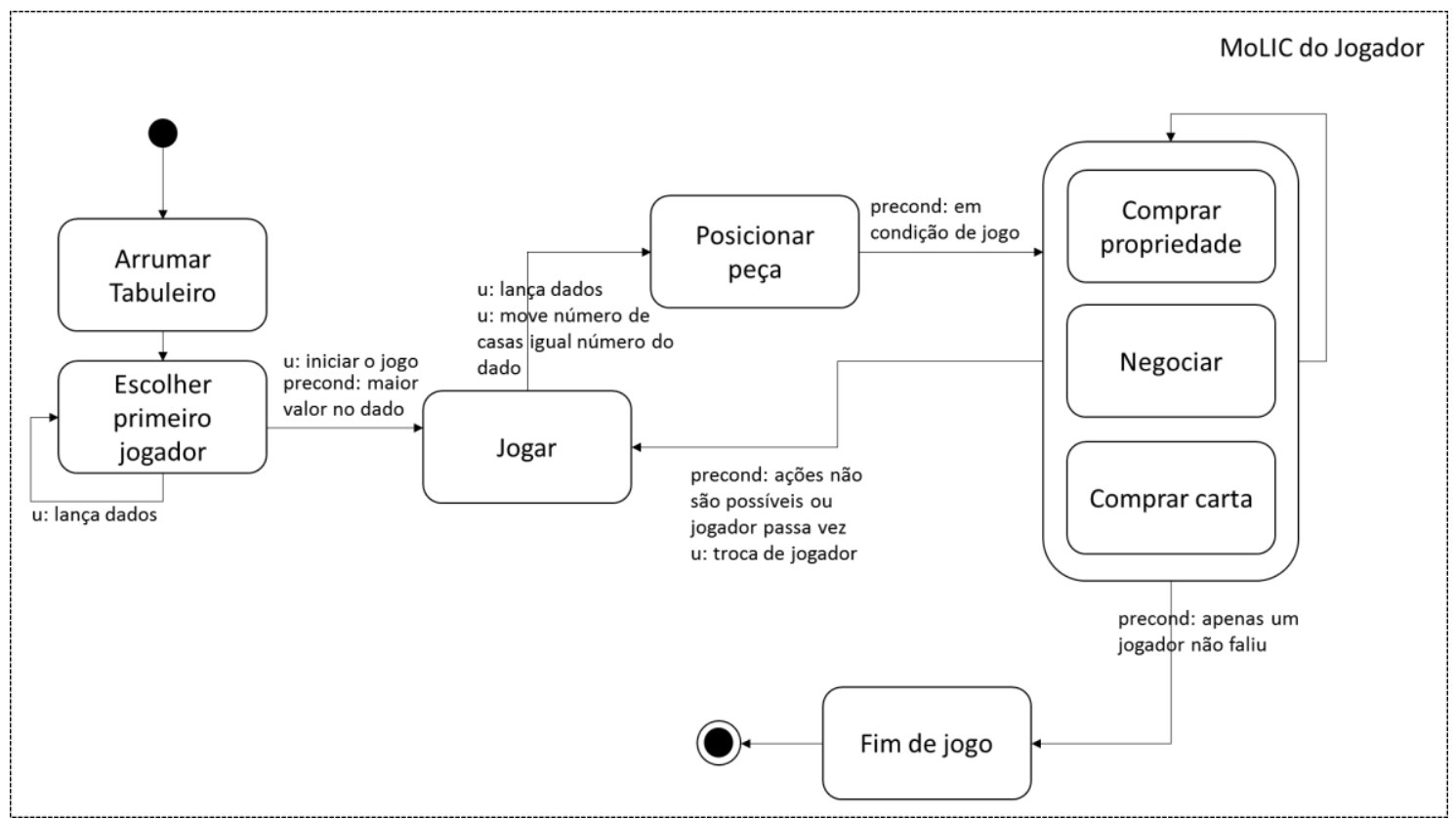

Figura 3-MoLIC do Monopoly ${ }^{\circledR}$.

Fonte: Elaborado pelo autor, com base na pesquisa realizada. 


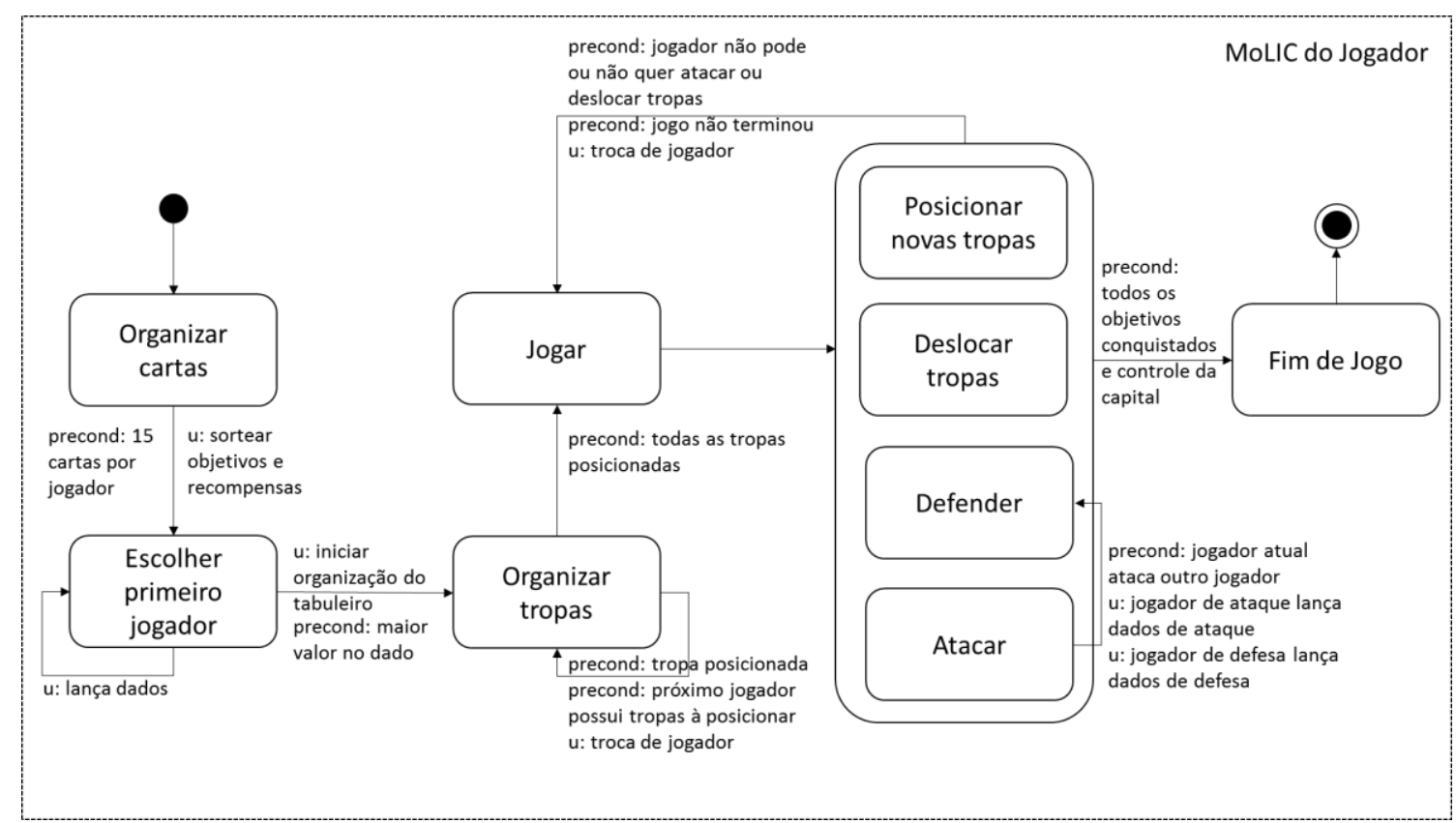

Figura 4 - MoLIC do Risk ${ }^{\circledast}$.

Fonte: Elaborado pelo autor, com base na pesquisa realizada.

\section{HEURÍSTICAS REDESENHADAS E A ANÁLISE}

Visibilidade de Status do Sistema: o tabuleiro, as regras e outros elementos do jogo devem sempre permitir ao usuário saber o que está acontecendo. (DESURVIRE et al, 2004) Isto significa que o usuário precisa saber de quem é o turno e/ou quais ações ele pode ou não executar em determinado momento.

O tabuleiro de Xadrez permite ao usuário saber em que posição cada peça, sua e do seu adversário está posicionada, permitindo aos jogadores antever suas jogadas nos turnos seguintes. O jogo em si permite facilmente a identificação dos turnos. Que fica ainda mais claro quando são utilizados os relógios de marcação. No Gamão essa identificação é tão clara quanto a do Xadrez, uma vez que o jogador que possui as peças brancas também só pode ficar em casas da respectiva cor. Além disso a separação de turnos é bem dividida pelo momento do lançamento dos dados. No jogo Monopoly as regras se mostram de fácil execução e o tabuleiro lúdico utiliza-se de cores para identificar grupos de propriedades onde poderão ser montadas casas ou hotéis. Além disso as peças representam objetos que os jogadores podem ter afinidade em seu cotidiano. $O$ Risk oferece um tabuleiro bem mais complexo que os outros jogos, com muitas peças e cartas a serem distribuídas aos jogadores. Além disso existe uma complexidade matemática na regra, o que pode dificultar aos jogadores saber exatamente e em pouco tempo o total de peças que ele deverá posicionar em cada turno.

Relacionamento entre a interface do sistema e o mundo real: o jogo deve falar a linguagem do usuário e não a linguagem técnica. Isto significa que mesmo que o jogo possua uma linguagem própria, essa linguagem deverá ser complementar à linguagem tradicional do usuário.

O jogo de Xadrez utiliza uma linguagem simples de ser entendida, já que os nomes das peças são adequados ao conhecimento comum do usuário. E mesmo possuindo uma linguagem própria como "cheque" ou "cheque mate", não é uma 
linguagem complicada para entendimento de um leigo. Por sua vez o Gamão enquanto facilita o relacionamento da interface com o mundo real ao oferecer termos como "casa livre" e "casa ocupada", também dificulta utilizando termos muito específicos do jogo como "blot" (para peças capturadas) e "checker" (para peças em jogo). Ambos os jogos Monopoly e Risk possuem termos totalmente específicos das suas regras, no entanto todos esses termos são analogias a ações e objetos do mundo real, como por exemplo "prisão" ou "impostos" no Monopoly e "tropas" ou "capital” no Risk.

Liberdade e controle do usuário: o usuário, sempre que desejar, deve poder desistir de uma ação, podendo voltar ao ponto anterior. O jogo não pode impedir uma operação do usuário, a não ser que essa operação possa gerar um erro, ou trapaça (PINELLE et al, 2008). Caso seja necessário executar uma determinada ação até o final do seu processamento sem interrupção, a regra do jogo deve informar ao usuário os motivos pelos quais a tarefa não pode ser cancelada.

As regras do Xadrez e do Gamão são bem específicas em relação a cada tipo de peça e seus movimentos, o jogador pode pensar a vontade antes de tocar em uma peça, no entanto, após tocá-la ele deverá executar algum movimento com a mesma, a não ser que o movimento seja impossível, além dos dados no caso do Gamão que não pode ser removido da mesa enquanto a jogada não terminar. No caso do Monopoly e do Risk algumas ações podem ser refeitas no mesmo turno (ou posteriormente) respeitando a regra do jogo, no entanto em determinadas situações essa liberdade pode gerar problemas pois as regras não definem o final do turno do jogador.

Consistência e Padronização: o jogo sempre deve utilizar o mesmo padrão de signos e palavras. Uma mesma ação sempre deve ter o mesmo efeito no jogo, independentemente de onde aconteça (DESURVIRE et al, 2004). Os códigos de cores e layout, de preferência, devem estar de acordo com os elementos reais conhecidos que possam estar inseridos no jogo.

Todos os quatro jogos estudados não modificam seu padrão de signos e palavras para peças, dados e tabuleiro. Mas nem todos os elementos são consistentes no que diz respeito a sua forma de utilização, a peça "cavalo" no Xadrez por exemplo, se move sob outras peças, e a contagem de novas tropas no Risk muda de acordo com o andamento do jogo, o que pode gerar alguma confusão em usuários iniciantes.

Prevenção de erros: deve-se criar mecanismos no jogo que possam prevenir os erros mais básicos do usuário. Para isto, utiliza-se dos conjuntos de regras e/ou mensagens através do tabuleiro ou de outros elementos do jogo antes de operações que possam alterar o jogo para um estado não adequado (por exemplo, uma trapaça ou uma situação que inviabilize os turnos seguintes) (PINELLE et al, 2008).

No jogo de Xadrez e no Gamão praticamente toda a prevenção de erros está disponível nas regras. Além disso, o jogador adversário (ou um possível juiz) faz toda a prevenção de erro, já que para ele não é interessante um movimento errado que beneficie seu adversário. O Monopoly e o Risk apresentam sua prevenção de erros tanto nas regras quanto nas cartas de jogo, que dizem exatamente o que deve ser feito pelo jogador, sem ambiguidades. Todos quatro jogos apresentam prevenção de erro na forma em que seus tabuleiros são organizados.

Reconhecimento e não lembrança: sempre que possível, evite que o usuário tenha que lembrar um conjunto muito grande de regras para efetuar uma determinada jogada. Recomenda-se um conjunto de $7+/-2$ regras como um número ideal para o jogo ou para um subconjunto do jogo (PINELLE et al, 2008). 
O jogo de Xadrez possui exatamente 6 tipos de peças e uma regra específica para cada uma delas. Além de regras gerais que se aplicam no todo. Existem ainda 3 regras muito específicas para peões, torre e rei. O que somadas atingem o número de 9 regras específicas importantes para o andamento do jogo. O Gamão possui para todo jogo um conjunto de 7 regras. Ambos Monopoly e Risk possuem um conjunto maior de regras, no entanto todas elas podem ser separadas em subconjuntos menores, o que facilita a curva de aprendizagem e a jogabilidade durante uma partida.

Flexibilidade e eficiência de uso: o jogo deve ser fácil para uso por usuários iniciantes, mas deve ser flexível para permitir que usuários avançados possam ter ganho de desempenho (DESURVIRE et al, 2004). Isto significa que, em um bom jogo, qualquer nível de usuário poderá usufruir do entretenimento com um grau de satisfação semelhante, e que qualquer usuário poderá tornar-se um usuário avançado à medida que se aprofunda nas regras do jogo.

Usuários iniciantes em todos os quatros jogos analisados precisam aprender as regras básicas de movimento, turno e ação. Essas regras permitem jogadores em fase inicial de aprendizado executarem o jogo a um nível satisfatório. Os jogos permitem também que qualquer jogador possa criar estratégias e situações de jogo com uma complexidade maior ao passo em que se aprofunda no jogo, além de permitir também que a cada jogo melhore suas habilidades.

Estética e design minimalista: o texto e o design do jogo devem ser sempre simples e objetivos. Deve-se evitar colocar na interface mais ou menos do que o usuário deve saber (DESURVIRE et al, 2004) (PINELLE et al, 2008).

Tanto para o Xadrez, quanto para o Gamão e o Monopoly, os jogos possuem elementos simples e objetivos. Com peças representando cada jogador, é fácil entender o papel de cada um no jogo. Além disso os espaços que as peças podem ocupar no tabuleiro são bem definidos. O Risk no entanto, por existir a possibilidade de muitas peças ocuparem o tabuleiro ao mesmo tempo, gera um excesso de informação que poderia ser resolvido com elementos numéricos ou outros simbolismos.

Ajudar os usuários a reconhecer, diagnosticar e sanar erros: as regras do jogo devem prever erros de forma passiva e simples, além de informar de forma correta ao usuário e indicar possíveis soluções claras (PINELLE et al, 2008). A mensagem de erro nunca pode intimidar ao usuário.

Todos os jogos possuem regras bem definidas que ao serem seguidas evitam erros desnecessários. No entanto apenas no Risk os erros são dificilmente corrigidos, mesmo com apenas um turno de diferença entre o momento do erro e o momento da percepção do erro.

Ajuda e documentação: um jogo eficiente deve ser tão fácil de utilizar que o usuário não precise de maior ajuda. Ainda assim, deve ser construído um bom conjunto de documentação das regras e situações exemplos para que a ajuda seja facilmente acessada pelo usuário em caso de dúvida (DESURVIRE et al, 2004) (PINELLE et al, 2008).

Todos os jogos possuem um bom conjunto de regras documentadas e de fácil acesso durante o jogo. No entanto não é possível utilizar nenhum deles sem as mesmas, ou sem um conhecimento prévio da mecânica de cada um. 
A partir dos estudos realizados foi possível montar uma relação entre os jogos do trabalho e as heurísticas redesenhadas como podemos observar no Quadro 1, entendendo assim como cada jogo atende cada heurística.

Quadro 1 - Relação entre os Jogos estudados e as Heurísticas Redesenhadas.

\begin{tabular}{|c|c|c|c|c|}
\hline Heurística Redesenhada & Xadrez & Gamão & Monopoly & Risk \\
\hline Visibilidade de Status do Sistema & Sim & Sim & Sim & Não \\
\hline $\begin{array}{l}\text { Relacionamento entre a interface } \\
\text { do sistema e o mundo real }\end{array}$ & Sim & Sim & Sim & Sim \\
\hline Liberdade e controle do usuário & Não & Não & Sim & Sim \\
\hline Consistência e Padronização & Não & Sim & Sim & Não \\
\hline Prevenção de erros & Sim & Sim & Sim & Sim \\
\hline $\begin{array}{l}\text { Reconhecimento e não } \\
\text { lembrança }\end{array}$ & Sim & Sim & Sim & Sim \\
\hline Flexibilidade e eficiência de uso & Sim & Sim & Sim & Sim \\
\hline Estética e design minimalista & Sim & Sim & Sim & Não \\
\hline $\begin{array}{l}\text { Ajudar os usuários a reconhecer, } \\
\text { diagnosticar e sanar erros }\end{array}$ & Sim & Sim & Sim & Não \\
\hline Ajuda e documentação & Sim & Sim & Sim & Sim \\
\hline Total de Heurísticas Atendidas & 8 & 9 & 10 & 6 \\
\hline
\end{tabular}

Fonte: Elaborado pelo autor, com base na pesquisa realizada

\section{CONCLUSÃO}

As heurísticas de Nielsen podem ser redesenhadas para atender sistemas não computacionais, desde que esses sistemas sejam modelados de forma a se assemelhar a um sistema computacional. O presente trabalho ilustra esse processo através da instanciação dessa técnica para a avaliação de usabilidade de jogos de tabuleiro nãodigitais.

O uso de modelos para esse processo é fundamental, dando ao especialista a noção real de como as interações do sistema acontecem. O MoLIC se mostra um ótimo apoio no planejamento da interação, facilitando a reflexão sobre a realização de atividades e a identificação de ações e problemas possíveis ao usuário do sistema. Sendo assim o MoLIC pode além de representar a interação homem-computador, representar a interação homem-máquina também como uma representação do diálogo travado entre usuário e sistema para completar uma tarefa.

Como trabalhos futuros pretende-se analisar a interação dos usuários com esses e outros jogos de tabuleiro, através de grupos de foco e observação. Além da proposta de uma categorização dos jogos de tabuleiro bem como uma modelagem padrão para cada categoria identificada. 


\section{REFERÊNCIAS}

BARBOSA, Simone Junqueira; SANTANA, Bruno. Interação Humano-Computador. Rio de Janeiro: Elsevier, 2010.

BASTIEN, J. M. Christian; SCAPIN, Dominique L. Evaluating a user interface with ergonomic criteria. In: International Journal of Human-Computer Interaction. Volume 7, Issue 2, 1995, P. 105-121.

BELMIRO, Arnaldo. Gamão: o Rei dos Jogos e o Jogo dos Reis, São Paulo: Ediouro, 1984.

DESURVIRE, Heather; CAPLAN, Martin; TOTH, Jozsef A. Using heuristics to evaluate the playability of games. In: $\mathrm{CHI}$ '04 Extended Abstracts on Human Factors in Computing Systems (CHI EA '04). New York, NY, USA: ACM, 2004. P. 1509-1512.

FEITOSA, Alexandre et. al. Definição Formal de Táticas de Xadrez por Meio da Autoria Incremental de Conceitos Heurísticos. In: Universidade Tecnológica Federal do Paraná-UTFPR / XVIII Simpósio Brasileiro de Informática na Educação - SBIE. Mackenzie, 2007.

HASBRO. Monopoly. Massachusetts, USA: Hasbro, 1999. JOGO DE TABULEIRO.

HASBRO. Risk. Massachusetts, USA: Hasbro, 1999. JOGO DE TABULEIRO.

JEFFRIES, Robin et. al. User interface evaluation in the real world: a comparison of four techniques. In: Proceedings of the SIGCHI Conference on Human Factors in Computing Systems (CHI '91). New York, NY, USA: ACM, 1991. P. 119-124.

MOLICH, Rolf; NIELSEN, Jakob. Improving a human-computer dialogue. Commun. Volume 33, Issue 3. ACM, 1990. P. 338-348.

NIELSEN, Jakob. Enhancing the explanatory power of usability heuristics. In: Proceedings of the SIGCHI Conference on Human Factors in Computing Systems (CHI '94). New York, NY, USA: ACM, 1994. P. 152-158.

NIELSEN, Jakob. Finding usability problems through heuristic evaluation. In: Proceedings of the SIGCHI Conference on Human Factors in Computing Systems (CHI '92).New York, NY, USA: ACM, 1992. P. 373-380.

NIELSEN, Jakob. Usability Engineering. San Francisco, CA, USA: Morgan Kaufmann Publishers Inc, 1993.

NIELSEN, Jakob. Usability inspection methods. In: Conference Companion on Human Factors in Computing Systems (CHI '94), New York, NY, USA: ACM, 1994. P. 413-414.

NIELSEN, Jakob; LANDAUER, Thomas K. A mathematical model of the finding of usability problems. In: Proceedings of the INTERACT '93 and CHI '93 Conference on Human Factors in Computing Systems (CHI '93). New York, NY, USA: ACM, 1993. P. 206-213.

NIELSEN, Jakob; MOLICH, Rolf. Heuristic evaluation of user interfaces. In: Proceedings of the SIGCHI Conference on Human Factors in Computing Systems (CHI '90). New York, NY, USA: ACM, 1990. P. 249-256.

PINELLE, David; WONG, Nelson; STACH, Tadeusz. Heuristic evaluation for games: usability principles for video game design. In Proceedings of the SIGCHI Conference on 
Human Factors in Computing Systems (CHI '08). New York, NY, USA: ACM, 2008. P. 1453-1462.

ZHANG, Z.; BASILI, V.; SHNEIDERMAN, B. Perspective-based Usability Inspection: An Empirical Validation of Efficacy. Empirical Software Engineering, 1999, P. 43-69. 